Feb. 1995

OS-GE 44-95

hep-ph/9502381

\title{
On the Phase of $B^{0}-\bar{B}^{0}$ Mixing Matrix Elements in the SUSY Standard Model
}

\author{
T. Kurimoto円 \\ Department of Physics, Faculty of Science, \\ Osaka University, \\ Machikaneyama 1-16, Toyonaka, Osaka 560, Japan
}

\begin{abstract}
It is shown that the complex phase of $B^{0}-\bar{B}^{0}$ mixing matrix element is same as that of the standard model one up to the minor correction of the order of $\left(m_{c} / m_{t}\right)^{2}$ or less in the SUSY standard model. This conclusion is valid as far as the Yukawa coupling constants are perturbative and realistic, and can be available for other realistic models where generation mixing and $\mathrm{CP}$ violation are solely due to Yukawa interaction among quarks and Higgs doublets.
\end{abstract}

\footnotetext{
${ }^{1}$ e-mail: krmt@phys.wani.osaka-u.ac.jp address after April 1995: Department of Physics, Faculty of Science,

Toyama University, Gofuku 3190, Toyama 930, Japan
} 
Investigation of $\mathrm{CP}$ violation phenomena in $B$ physics is very important not only for fixing the parameters in the standard model but also for exploring new physics beyond the standard model. [1] $\mathrm{CP}$ violation in $B$ decay can occur through the interference between $B^{0}-B^{0}$ mixing and a decay amplitude as in the case of decay rate asymmetry in neutral $B$ meson decay into a CP eigenstate like $J / \psi K_{s}$. There, the complex phase of the $B^{0}-\bar{B}^{0}$ mixing matrix element, $M_{12}=\left\langle B^{0}\left|\mathcal{H}^{\Delta B=2}\right| \bar{B}^{0}\right\rangle$, is significant since the asymmetry is proportional to $\operatorname{Im}[(q / p) \rho]$, where

$$
\frac{q}{p} \cong \frac{M_{12}^{*}}{\left|M_{12}\right|}, \quad \rho \equiv \frac{A m p\left[\bar{B}^{0} \rightarrow f_{C P}\right]}{A m p\left[B^{0} \rightarrow f_{C P}\right]},
$$

in the $B$ meson case. The standard model gives [2]

$$
\arg \left[M_{12}\right] \cong \arg \left[\left(V_{t d}^{*} V_{t b}\right)^{2}\right]
$$

where $V_{i j}$ is the Kobayashi-Maskawa matrix element. [3] If a new physics contributes significantly to $B^{0}-\bar{B}^{0}$ mixing, the mixing matrix element $M_{12}$ is different from the standard model value. The discrepancy between the standard model prediction and the experimental data can shed light on new physics search. The magnitude of $M_{12}$ is related to the oscillation frequency of $B^{0}-\bar{B}^{0}$ mixing or the mass difference between two mass eigenstates, $\Delta M_{B}$, while the phase to $\mathrm{CP}$ violation asymmetry. The mass difference has been already measured, and the CP asymmetry will be within our reach by the $B$ dedicated experimental facilities now under construction. [4] Investigation of the prediction on $B$ physics in models beyond the standard model is now called for the search of new physics at those $B$ experiments.

Here it is shown that the phase of $M_{12}$ is same as the standard model value in the models which satisfy these conditions;

1. CP violation and generation mixing are solely originate from Yukawa couplings among quarks and Higgs $S U(2)_{L}$ doublets.

2. Yukawa coupling constants are perturbative and realistic.

3. Quarks are given in 3 generations.

4. Flavor changing neutral current is forbidden at the tree level. (Natural flavor conservation [5]) 
As a concrete example we first investigate SUSY standard model, which satisfies above conditions. Then we extend the results to other models like multiHiggs models where the above conditions are satisfied. The CP asymmetry is same as the standard prediction but the magnitudes of $B^{0}-\bar{B}^{0}$ mixing can differ in these models. Then the so called unitarity triangle[1] made from angles alone and that from sides alone are not consistent with each other, which is a sign of new physics.

It has been shown that the SUSY standard model gives the $B^{0}-\bar{B}^{0}$ mixing matrix elements $M_{12}$ of the same phase as that of the standard model one in the case where top quark Yukawa coupling is far larger than other Yukawa couplings. [6]. Recently this is found to be the case even if top quark Yukawa coupling is not so larger than bottom quark Yukawa coupling as far as the Yukawa couplings are perturbative and realistic by Goto, Nihei and Okada's numerical computation. [7] Here we give an analytic explanation of it.

SUSY standard model has 2 Higgs doublets, $H$ and $H^{\prime}$, which couple to quark fields as

$$
\mathcal{L}_{\text {Yukawa }}=\overline{D_{R}} y_{D} Q_{L} H+\overline{U_{R}} y_{U} Q_{L} H^{\prime}+\text { h.c. },
$$

where $Q_{L}=(U, D)_{L}$, and we abbreviated 3 generation quark fields by $U=$ $(u, c, t)$ and $D=(d, s, b)$. The Yukawa coupling constants, $y_{D}$ and $y_{U}$, are $3 \times 3$ complex matrices. The key point of the following argument lies in the fact that generation changing interaction is solely controlled by the above Yukawa interaction (condition 1). It is also important to pay attention to the fact that left-handed quark fields always come right-side of the Yukawa couplings while right-handed fields to left-side. Without loss of generality we can take the basis where $y_{D}$ is real positive diagonal and $y_{U}$ is expressed in terms of up-type quark masses and Kobayashi-Maskawa matrix $V$ (condition $4)$;

$$
\begin{aligned}
& y_{D}=\operatorname{diag}\left(m_{d}, m_{s}, m_{b}\right) /\left\langle H^{0}\right\rangle \equiv \tilde{M}_{D} \\
& y_{U}=\operatorname{diag}\left(m_{u}, m_{c}, m_{t}\right) V /\left\langle H^{\prime 0}\right\rangle \equiv \tilde{M}_{U} V
\end{aligned}
$$

The coupling among quarks and $W$ boson is still generation diagonal in this basis. Then any generation mixing in the theory should be expressed in terms of the Yukawa couplings (condition 2). For example, the d-type scalar quark 
mass matrix,

$$
\left(\begin{array}{cc}
\tilde{D}_{L}^{\dagger} & \tilde{D}_{R}^{\dagger}
\end{array}\right)\left[\begin{array}{cc}
(L-L)_{D} & (L-R)_{D} \\
(L-R)_{D}^{\dagger} & (R-R)_{D}
\end{array}\right]\left(\begin{array}{c}
\tilde{D}_{L} \\
\tilde{D}_{R}
\end{array}\right)
$$

is expanded in the Yukawa couplings in the following way;

$$
\begin{aligned}
(L-L)_{D} & =m_{D L}^{2} I+\sum_{\mathbf{p}=\left(p_{1}, p_{2}, \ldots\right)} \kappa_{D L}^{(\mathbf{p})}\left(y_{D}^{\dagger} y_{D}\right)^{p_{1}}\left(y_{U}^{\dagger} y_{U}\right)^{p_{2}} \cdots\left(y_{D}^{\dagger} y_{D}\right)^{p_{j}} \\
& =m_{D L}^{2} I+\sum_{\mathbf{p}=\left(p_{1}, p_{2}, \ldots\right)} \kappa_{D L}^{(\mathbf{p})} \tilde{M}_{D}^{2 p_{1}}\left(V^{\dagger} \tilde{M}_{U}^{2 p_{2}} V\right) \cdots \tilde{M}_{D}^{2 p_{j}}, \\
(L-R)_{D} & =m_{D L R}^{2} I+\sum_{\mathbf{p}=\left(p_{1}, p_{2}, \ldots\right)} \kappa_{D L R}^{(\mathbf{p})} \tilde{M}_{D}^{2 p_{1}}\left(V^{\dagger} \tilde{M}_{U}^{2 p_{2}} V\right) \cdots \tilde{M}_{D}^{2 p_{j}} \tilde{M}_{D}, \\
(R-R)_{D} & =m_{D R}^{2} I+\sum_{\mathbf{p}=\left(p_{1}, p_{2}, \ldots\right)} \kappa_{D R}^{(\mathbf{p})} \tilde{M}_{D} \tilde{M}_{D}^{2 p_{1}}\left(V^{\dagger} \tilde{M}_{U}^{2 p_{2}} V\right) \cdots \tilde{M}_{D}^{2 p_{j}} \tilde{M}_{D} .
\end{aligned}
$$

The index $p_{i}$ takes a value of non-negative integer. The coefficients $\kappa^{(\mathbf{p})}$ and the generation diagonal parts are constants fixed in the model. Note the order of Yukawa couplings given in these formulae as pointed out below Eq.(3).

We calculate the contribution to $B^{0}-\bar{B}^{0}$ mixing by treating the generation mixing as a perturbation (mass insertion). The gauge interactions are all generation diagonal in our basis. The generation mixing part of the gluino contribution to $b \rightarrow d$ transition shown in Fig.1 is given as

$$
\left[\tilde{M}_{D}^{r_{1}}\left(V^{\dagger} \tilde{M}_{U}^{2 r_{2}} V\right) \tilde{M}_{D}^{r_{3}} \cdots\left(V^{\dagger} \tilde{M}_{U}^{2 r_{n-1}} V\right) \tilde{M}_{D}^{r_{n}}\right]_{13}
$$

By using the hierarchy among quark masses

$$
m_{t} \gg m_{c} \gg m_{u}, \quad m_{b} \gg m_{s} \gg m_{d}
$$

and that among Kobayashi-Maskawa matrix elements (conditions 2 and 3),

$$
V=O\left(\begin{array}{ccc}
1 & \lambda & \lambda^{3} \\
\lambda & 1 & \lambda^{2} \\
\lambda^{3} & \lambda^{2} & 1
\end{array}\right), \quad\left(\lambda=\left|V_{u s}\right|\right)
$$


we find that the dominant contribution is given by

$$
\begin{aligned}
& \left(\tilde{M}_{D}^{r_{1}}\right)_{11}\left(V^{\dagger}\right)_{13}\left(\tilde{M}_{U}^{2 r_{2}}\right)_{33}(V)_{33} \cdots\left(V^{\dagger}\right)_{33}\left(\tilde{M}_{U}^{2 r_{n-1}}\right)_{33}(V)_{33}\left(\tilde{M}_{D}^{r_{n}}\right)_{33} \\
\propto & m_{d}^{r_{1}} m_{t}^{p} m_{b}^{q}\left|V_{33}\right|^{2 s}\left(V^{\dagger}\right)_{13} V_{33}=m_{d}^{r_{1}} m_{t}^{p} m_{b}^{q}\left|V_{t b}\right|^{2 s} V_{t d}^{*} V_{t b} .
\end{aligned}
$$

and the next leading part is suppressed by $\left(m_{c} / m_{t}\right)^{2}$. This situation is shown schematically in Fig.2. Therefore we get

$$
\arg \left[M_{12}^{\text {gluino }}\right] \cong \arg \left[\left(V_{t d}^{*} V_{t b}\right)^{2}\right] \cong \arg \left[M_{12}^{S M}\right] .
$$

Similar calculation of chargino, neutralino and charged Higgs contributions give the same conclusion although the calculation is a bit complicated.

Now we give a simpler explanation of the above result. We have at most 4 types of $b \rightarrow d$ transition effective operators under the conditions 1 and 2 ;

$$
\begin{array}{ll}
\overline{d_{L}} \Gamma\left[y^{\dagger} \cdots y\right]_{13} b_{L}, & \overline{d_{R}} \Gamma\left[y_{D}\left(y^{\dagger} \cdots y\right)\right]_{13} b_{L}, \\
\overline{d_{L}} \Gamma\left[\left(y^{\dagger} \cdots y\right) y_{D}^{\dagger}\right]_{13} b_{R}, & \overline{d_{R}} \Gamma\left[y_{D}\left(y^{\dagger} \cdots y\right) y_{D}^{\dagger}\right]_{13} b_{R},
\end{array}
$$

where $\Gamma$ is a combination of $\gamma$ matrices, and $y=y_{D}$ or $y_{U}$. So the leading coefficient of the above terms is given by

$$
m_{d}^{r} m_{t}^{p} m_{b}^{q}\left|V_{t b}\right|^{2 s} V_{t d}^{*} V_{t b}
$$

in our basis of Yukawa coupling under the conditions 3 and 4 . We find that

$$
\left.M_{12}=\left\langle B^{0}\left|f(\bar{d} \Gamma b)\left(\bar{d} \Gamma^{\prime} b\right)\right| \bar{B}^{0}\right\rangle \cong\left(V_{t d}^{*} V_{t b}\right)^{2} \times \text { (real factor }\right) .
$$

This conclusion holds as far as the model satisfies the four conditions given before because we have made no model specific calculation.

Before closing this letter we give one comment on the power of quark masses in $M_{12}$. The standard model also satisfies the four conditions. If we calculate $M_{12}$ treating the generation changing by mass insertion method as given before in the SUSY standard model case, the contribution shown in Fig.3 gives $M_{12} \propto m_{t}^{4}\left(V_{t d}^{*} V_{t b}\right)^{2}$. But we know that $M_{12} \propto m_{t}^{2}\left(V_{t d}^{*} V_{t b}\right)^{2}$ in the ordinary calculation if $m_{t} \ll M_{W}$. 8 . This mystery is solved once we include all the remaining contributions of mass insertion. The exact formula of $M_{12}$ in the standard model depends on quarks masses as follows;

$$
\begin{aligned}
& \xi_{x} \xi_{y}\left[-\frac{3}{4} x y+\frac{x^{2} \ln x-y^{2} \ln y}{x-y}+O\left(m_{q}^{6}\right)\right] \\
\rightarrow & \xi_{x}^{2}\left[-\frac{3}{4} x^{2}+x+2 x \ln x+O\left(m_{q}^{6}\right)\right] \quad(y \rightarrow x),
\end{aligned}
$$


where $\xi_{i}=V_{i d}^{*} V_{i b}$ and $x=\left(m_{x} / M_{W}\right)^{2}$. The numerator of the second term of Eq.(16) is $O\left(m_{q}^{4}\right)$. Inclusion of full mass-insertion contribution gives the denominator $x-y$, and the $\ln$ term appears if careful treatment of infrared divergence is done in the calculation. In any case the phase of $M_{12}$ is controlled by $\left(V_{t d}^{*} V_{t b}\right)^{2}$ because $\left|m_{t}^{n}\left(V_{t d}^{*} V_{t b}\right)^{2}\right| \gg\left|m_{c}^{n}\left(V_{c d}^{*} V_{c b}\right)^{2}\right|$ as far as $n \geq 1$.

In conclusion we have shown that the phase of the $B^{0}-\bar{B}^{0}$ mixing matrix element is unique in the models where generation mixing and $\mathrm{CP}$ violation solely originate from the naturally flavor conserving Yukawa coupling among 3 generations of quarks and Higgs doublets as far as the Yukawa coupling constants are realistic and perturbative. Examples of the models which satisfy these criterions are the standard model, multi-Higgs models with NFC and the SUSY standard model. We need informations both of sides and angles of the unitarity triangle to find a signal of these models at coming $B$ factories.

\section{Acknowledgment}

The author would like to thank Professor Y. Okada at KEK for fruitful discussions. This work is supported in part by Grant-in Aids for Scientific Research from the Ministry of Education, Science and Culture (No. 06740218 and 05228104). 


\section{References}

[1] For reviews, see

I.I. Bigi, V.A. Khoze, N.G. Uraltsev, and A.I. Sanda, in CP Violation, ed. C. Jarlskog (World Scientific, Singapore, 1989);

Y. Nir, in Proc. of the 20th SLAC Summer Institute on Particle Physics, ed. L. Vassilian, SLAC-REPORT-412 (1992);

J.L. Rosner, in B DECAYS revised 2nd edition, ed. S. Stone (World Scientific, Singapore, 1995);

Y. Nir and H. Quinn, ibid.

[2] H.Y. Cheng, Phys. Rev. D26, 143 (1982);

A.J. Buras, W. Slominsky and H. Steger, Nucl. Phys. bf B245, 369 (1984).

[3] M. Kobayashi and T. Maskawa, Prog.Theor.Phys. 49, 652 (1973).

[4] For a review, see M. Artuso, in B DECAYS revised 2nd edition, ed. S. Stone (World Scientific, Singapore, 1995).

[5] G.C. Branco, Phys. Rev. Lett. 44, 504 (1980).

[6] J.-M. Gérard, W. Grimus, A. Masiero, D.V. Nanopoulos, and A. Raychaudhuri, Nucl. Phys. B253, 93 (1985);

M. Dugan, B. Grinstein, and L. Hall, Nucl. Phys. B255, 413 (1985);

G. Altarelli and P. Franzini, Z. Phys. C37, 241 (1988);

T. Kurimoto, Phys. Rev. D39, 3447 (1989).

[7] Y. Okada, preprint KEK-TH-428, KEK Preprint 94-192, hepph/9502240, February, 1995.

[8] T. Inami and C.S. Lim, Prog.Theor.Phys. 65, 297 (1981). 


\section{Figure Captions}

Fig.1 Gluino contribution to $b \rightarrow d$ transition. The black circle represents a scalar quark mass matrix element. The indices $j, k$ and $l$ specify scalar quarks.

Fig.2 Diagrammatic estimation of the matrix element (8). The number at the left side represents generation. Transition between 1st and 2nd (2nd and 3rd) generations gets $O(\lambda)\left(O\left(\lambda^{2}\right)\right)$ factor from quark mixing matrix $V$. A factor of a power of quark mass is multiplied at each circle. At least the factor of $O\left(\lambda^{3}\right)$ is necessary in $b \rightarrow d$ transition. The dominant contribution is obtained by multiplying as least 1 st or 2nd quark masses as possible.

Fig.3 A standard model contribution to $B^{0}-\overline{B^{0}}$ mixing calculated in the mass insertion scheme taken here. 

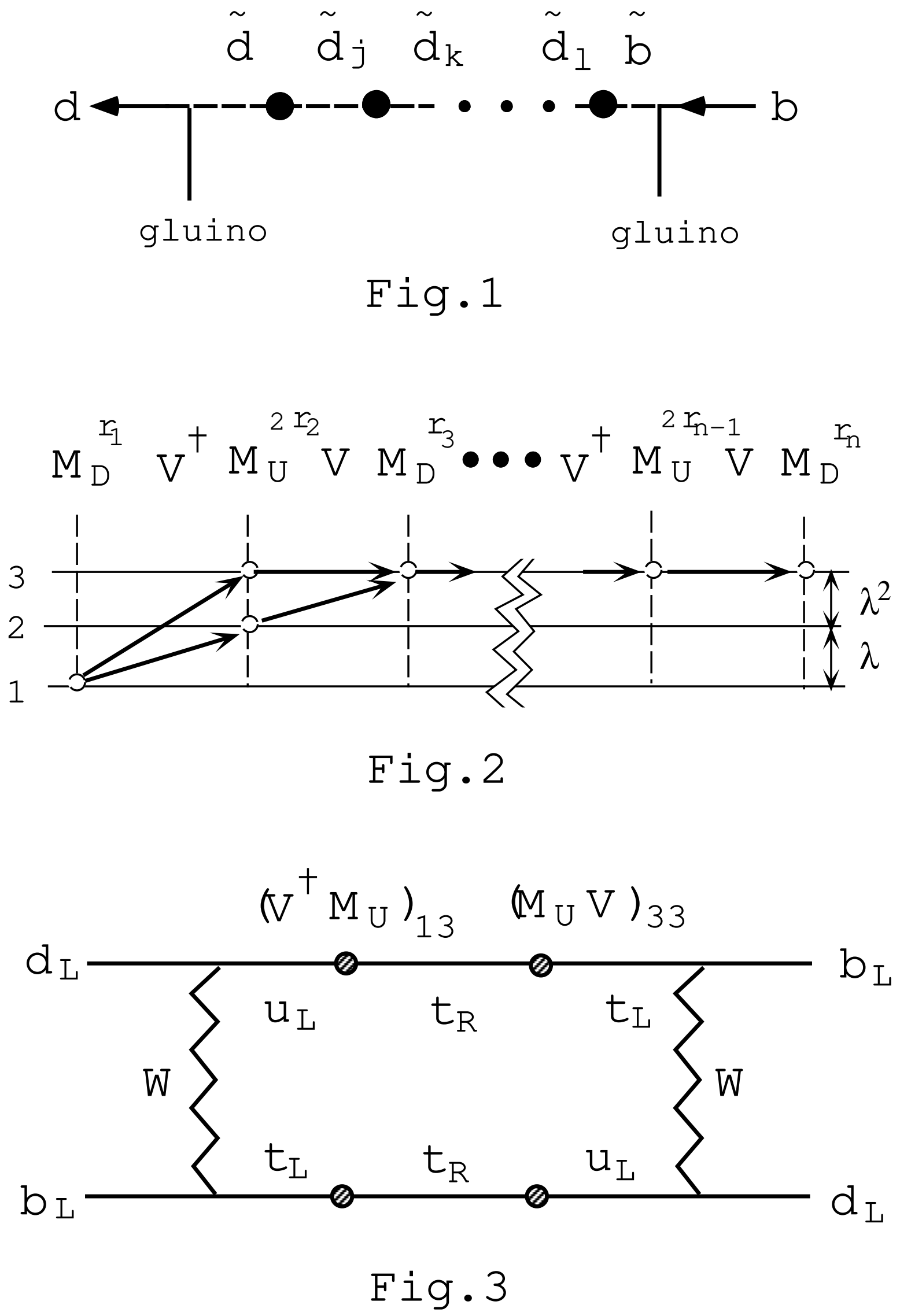